\title{
Intrathecal baclofen pump implantation for complex regional pain syndrome in a patient with a spinal cord stimulator: consideration about optimal location of intrathecal catheter tip - A case report -
}

Received September 20, 2017

Revised 1st, October 23, 2017

2nd, October 30, 2017

Accepted October 30, 2017

\section{Corresponding author}

Sang Eun Lee, M.D.

Department of Anesthesiology

and Pain Medicine, Inje University

Haeundae Paik Hospital, 875

Haeun-daero, Haeundae-gu, Busan

48108, Korea

Tel: 82-51-797-0425

Fax: 82-51-797-0499

E-mail: anelse@naver.com

ORCID

http://orcid.org/0000-0002-9029-4991

\section{Myoung Jin Ko ${ }^{1}$, Hyun-seong Lee', Hyunji Jo', Seong Rok Kim², Sangyoon Jeon ${ }^{3}$, and Sang Eun Lee ${ }^{1}$}

Department of Anesthesiology and Pain Medicine, 'Inje University Haeundae Paik Hospital, Busan, ${ }^{2}$ The Keon Sarang Medical Office, Geoje, ${ }^{3}$ Dong Kang Hospital, Ulsan, Korea

\begin{abstract}
Intrathecal baclofen (ITB) pump implantation can be used to control dystonia and severe pain associated with complex regional pain syndrome (CRPS) with or without a spinal cord stimulator (SCS). A 45-year-old female patient had gotten an SCS to control the pain of CRPS. However, she suffered from chronic intractable pain in her left ankle and foot despite paresthesia in the entire painful area because the effectiveness of the SCS gradually diminished over time. In a trial of intrathecal drug administration, baclofen was superior to morphine for pain relief, had fewer side effects, and was superior in terms of patient satisfaction. To achieve the greatest degree of pain relief from the ITB pump, the tip of the intrathecal catheter was carefully placed in relation to the SCS. Over a oneyear follow-up period, the patient experienced mild pain without any adverse effects.
\end{abstract}

Key Words: Baclofen, Complex regional pain syndrome, Intrathecal pump, Spinal cord stimulation.
Complex regional pain syndrome (CRPS) is a chronic pain condition that most often affects one of the limbs, generally after an injury or trauma to that limb; it is characterized by pain and sensitivity, swelling, temperature and skin changes, and motor dysfunction in the affected area [1]. CRPS is associated with dysregulation of the central nervous system (CNS), peripheral nervous system, and autonomic nervous system. The pathophysiology and best course of treatment for CRPS are undetermined, and treatment is evolving. A spinal cord stimulator (SCS) is considered a management tool for CRPS patients who have uncontrolled pain despite use of medication or other interventional therapies. However, the effectiveness of an SCS decreases over time and unsatisfactory pain relief is often seen in CRPS patients even though the SCS cov- ers the painful region well.

Intrathecal baclofen (ITB) pumps have been used to ameliorate global pain in patients with CRPS and dystonia based on the anti-nociceptive effects of ITB [2-5]. Furthermore, several studies have reported the clinical effectiveness of combined SCS and ITB pump therapy as a multimodal therapeutic approach [3,5]. These studies illustrate that ITB therapy can enhance the effect of an SCS, even though ITB therapy alone does not produce sufficient pain relief. Additionally, they did not describe the importance of the location of the intrathecal catheter, which delivers a very small dose of medication at a very slow rate of infusion. We discuss a clinical experience with ITB pump implantation in a patient with advanced CRPS with an implanted SCS, focusing on the

This is an Open Access article distributed under the terms of the Creative Commons Attribution Non-Commercial License (http://creativecommons.org/licenses/by-nc/4.0) which permits unrestricted non-commercial use, distribution, and reproduction in any medium, provided the original work is properly cited. 
importance of the position of the intrathecal catheter.

\section{CASE REPORT}

A 45-year-old woman was referred to our pain clinic complaining of severe pain in her left foot after surgery for Hallux valgus. The patient had undergone foot surgery eight times. The surgical procedures were likely to be related to an injury to the left superficial peroneal nerve. Electromyography and nerve conduction test revealed that the patient had autonomic dysfunction and left superficial peroneal neuropathy. Digital infrared thermal imaging (DITI) revealed a significant difference in temperature between the two legs. There was increased uptake in the left ankle and foot visible on a threephase bone scan, which also coincided with CRPS. The patient was diagnosed with type 2 CRPS. Her pain was characterized by burning, stabbing, and pricking. The patient's visual analogue scale (VAS) score was above 70/100 (0, no pain; 100, worst possible pain). The pain was refractory to pharmacological and interventional treatments. The patient was given a permanent SCS (Restore Sensor, Medtronic Inc., USA), with good trial effectiveness. The sweet spot of the SCS was the 3rd lead of the octrode in the thoracic vertebra at level 11 and the pain in the left ankle area was well covered. During the first month, the patient's VAS score decreased to 30. However, the effect of SCS decreased over time. After 3 months of SCS implantation, the burning sensation and allodynia were getting worse, and reached a VAS score of 90 despite multiple pharmacologic treatments and interventional blocks, so it was very uncomfortable for her to walk. She also complained of sleep disturbances and weakness of the left leg. After 6 months, intrathecal morphine administration was attempted for pain control. However, the patient complained of unsatisfactory pain relief and nausea. We also tried to inject baclofen intrathecally. The test dose of baclofen was 50 $\mu \mathrm{g}$ in $1 \mathrm{ml}$. For a day and a half, the pain was reduced to a VAS score of 20 , but the patient complained of whole body sweating and nausea. The next day, she was more comfortable because her pain level was much reduced and the temporary nausea had disappeared.

We decided to continuously infuse 50-100 $\mu \mathrm{g} /$ day of intrathecal baclofen for about 10 days using a catheter $(20 \mathrm{G}$, BD Perisafe; BD Medical, USA), and the baclofen was infused intrathecally at $50 \mu \mathrm{g}$ per day using a GemStar patientcontrolled analgesia pump (Hospira Inc., USA). When baclofen was infused at $75 \mu \mathrm{g} / \mathrm{day}$, the patient's VAS score was reduced to 25 from 80, but mild nausea was observed. When baclofen was infused at $100 \mu \mathrm{g} / \mathrm{day}$, the VAS score did not decrease any further and moderate to severe nausea was observed. On the basis of these results, we decided to permanently implant a Medtronic SynchroMed II programmable infusion system for intrathecal drug delivery. Approaching
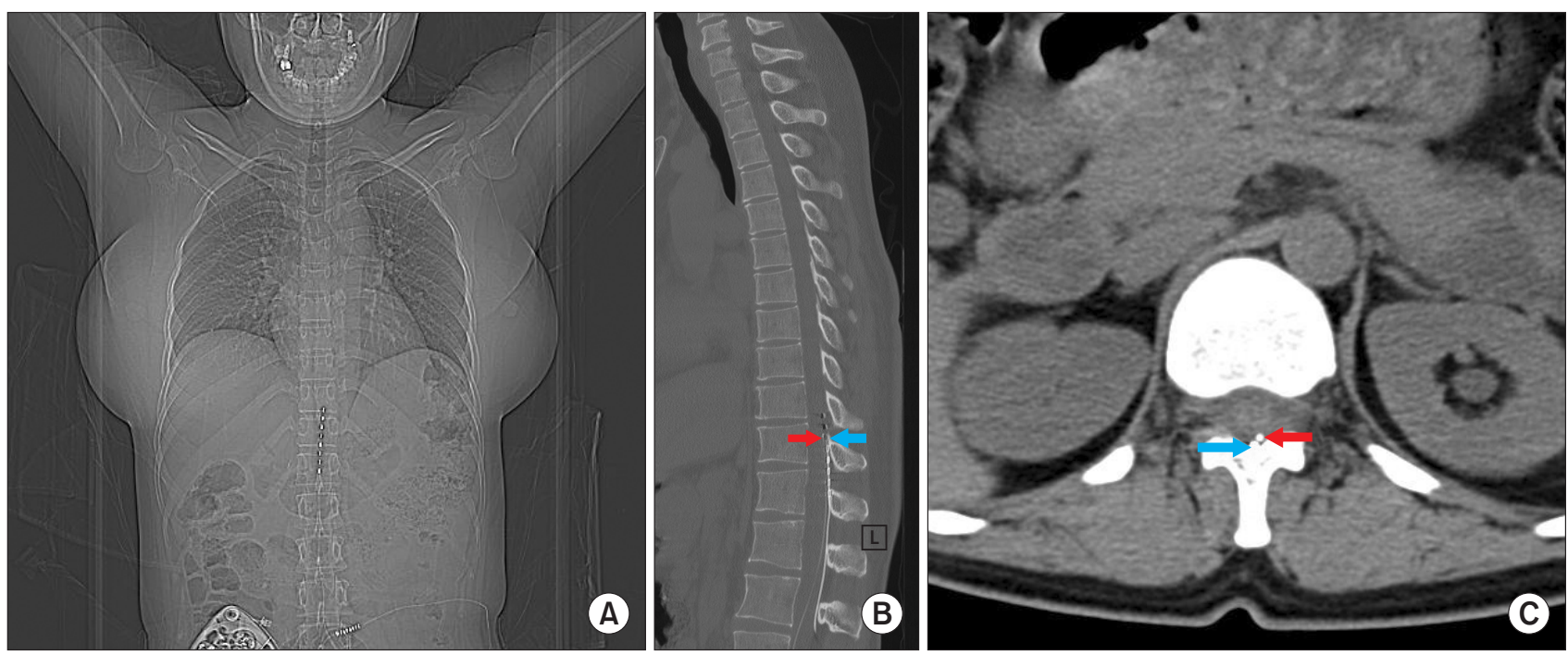

Fig. 1. (A) Computed tomography showed the intrathecal catheter tip is located at same level with spinal cord stimulator's 3rd lead (sweet spot of spinal cord stimulator) in thoracic vertebra 11. (B, C) The tip of intrathecal catheter (white arrow) is placed in ipsilateral (left) dorsal subarachnoid space at same level with the sweet spot (3rd lead) of spinal cord stimulator (black arrow) in thoracic vertebra 11 level. 
from lumbar vertebrae 3-4, the tip of an Ascenda intrathecal catheter (Medtronic, USA) was placed at the sweet spot of the SCS in the thoracic vertebra at level 11 (Fig. 1), assuming that the target sites in the spinal cord for baclofen would be near the sweet spot of the SCS. A SynchroMed implantable pump
(Medtronic, USA) was inserted into the patient's left lower abdomen. The pump was filled with normal saline $(5 \mathrm{ml})$ and highly concentrated baclofen ( $15 \mathrm{ml})$ ( $1 \mathrm{mg} / \mathrm{ml})$. Referring to the trial results, intrathecal baclofen was infused at $72 \mu \mathrm{g} /$ day and the total daily volume was $0.096 \mathrm{ml}$. The patient's VAS
A
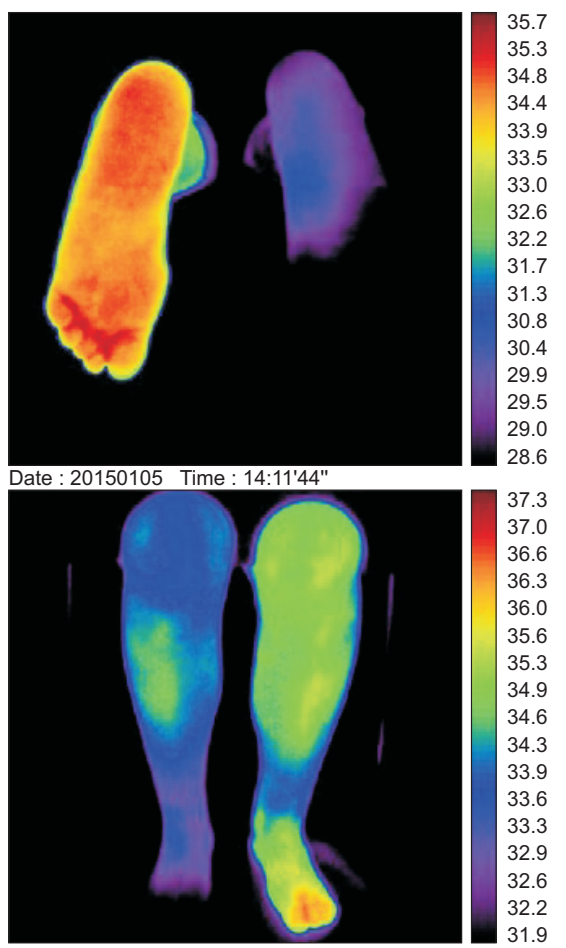

B
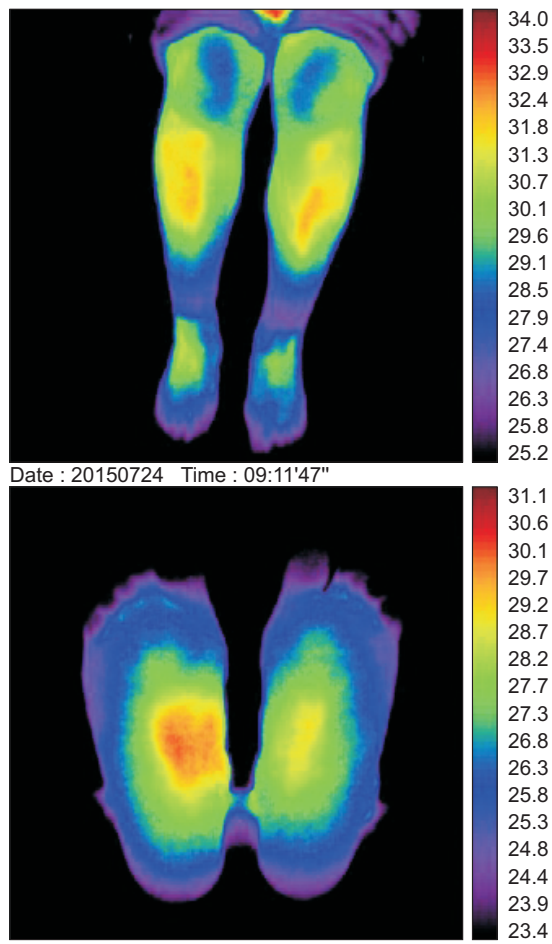
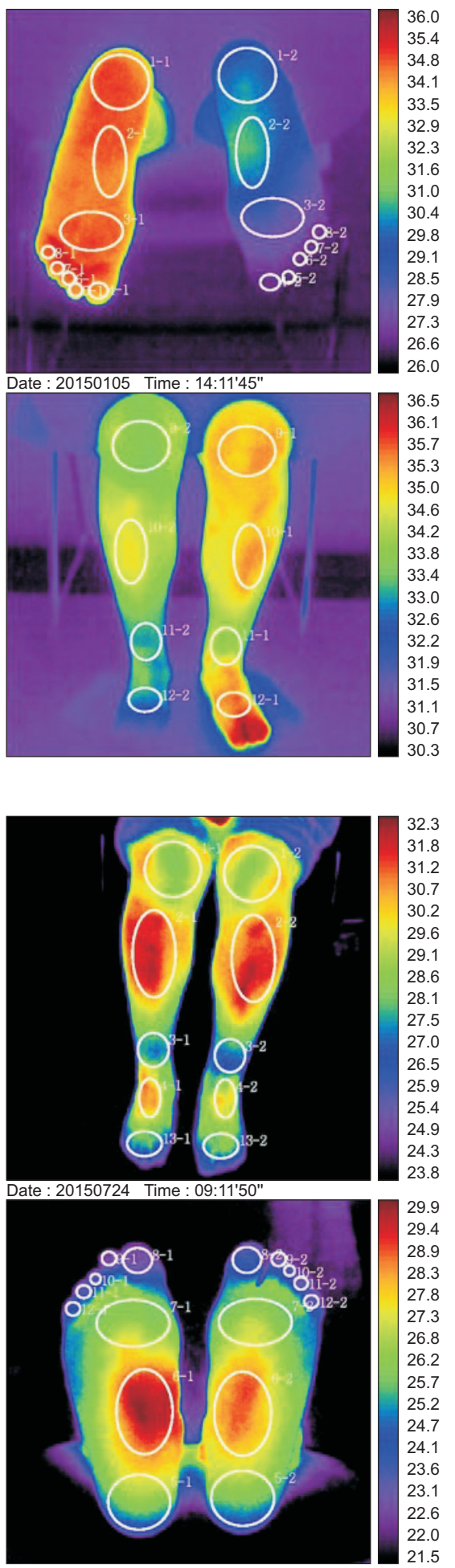

Fig. 2. Digital infrared thermal imaging before and after intrathecal baclofen (ITB) pump implantation. (A, before) There were significant temperature differences more than $2^{\circ} \mathrm{C}$ between both ankle and foot before ITB pump implantation. (B, after) Symmetric temperature pattern was observed between both ankle and foot after ITB pump implantation. 
score was reported as 15 . She had an intermittent headache on postoperative day (POD) 3, which disappeared over time. On POD 5, serous fluid leaked around the suture site, and this gradually worsened, so surgical debridement was performed to promote wound healing. Three months after intrathecal pump system implantation, DITI showed a normal temperature distribution between the legs as compared with the previous image (Fig. 2) In addition, the number of drugs taken for pain management was gradually reduced and, finally, the patient stopped all medications 45 days after the surgery and returned to daily life, except that she continued to take pregabalin 75-150 mg per day intermittently. At a 12-month followup, although the dose of baclofen was increased to $100 \mu \mathrm{g} /$ day, she had little pain (VAS score 10) and no side effects. The relative contributions to pain relief that the patient expressed were as follows: intrathecal baclofen, $60 \%$; SCS, $25 \%$; medication, $15 \%$. The pain was well controlled without significant increases even though the SCS was turned off. The patient was satisfied with ITB pump implantation because she was able to return to her social life.

\section{DISCUSSION}

In this case, we saw a dramatic reduction of pain in a CRPS patient following intrathecal baclofen pump implantation without significant complications. CRPS is a disabling disease that is difficult to treat. The pathophysiology of CRPS is not completely understood. However, several mechanisms have been proposed to contribute to this disease, including inflammation, vasomotor dysfunction, central sensitization, and neuroplasticity [6]. Some CRPS patients experience intractable pain which is unresponsive to conventional therapies, such as medications, physical therapy, and nerve block. In such patients, SCS may be a good treatment option. The use of an SCS as a pain control measure for CRPS patients is supported by a good deal of evidence $[7,8]$. However, some patients have unresponsive pain despite use of an SCS. Furthermore, some patients complain of a reduction in the analgesic effect over time. For patients who experience unsatisfactory pain reduction with an SCS, intrathecal drug administration can be considered. Morphine is the most widely used intrathecally administered drug for pain control [9]. However, in our case, the patient had side effects such as nausea and dizziness when administered intrathecal morphine. Compared with morphine administration, ITB produces great pain reduction without significant side effects such as motor weakness. In our experience, ITB causes male patients discomfort with urinary dysfunction and impotence, requiring medication. Therefore, as morphine appears to have less of an analgesic effect or is associated with significant side effects, intrathecal baclofen can be considered as an alternative.

Baclofen is a $\gamma$-aminobutyric acid $(\mathrm{GABA})_{\mathrm{B}}$ receptor agonist. $\mathrm{GABA}_{\mathrm{B}}$ agonists act by increasing potassium conductance, thus producing membrane hyperpolarization. In addition, $\mathrm{GABA}_{\mathrm{B}}$ agonists cause inhibition of calcium conductance across voltage-gated $\mathrm{Ca}^{2+}$ channels and decrease the evoked release of excitatory neurotransmitters. Also, baclofen can attenuate pain perception by affecting pain transmission systems, suppress neuropathic pain, down-regulate the sympathetic nervous system, and decrease pain associated with muscle spasms [4]. Intrathecal infusion of baclofen has been used to treat spasticity since the 1980s [10]. The antinociceptive effects of intrathecal baclofen administration were identified in an animal study [2]. Moreover, several human studies reported that intrathecal baclofen is effective for chronic pain or neuropathic pain [3-5].

Neuropathic pain patients suffering from incomplete pain control with an SCS can be provided intrathecal baclofen injections. The effect of an SCS on neuropathic pain may be improved by intrathecal baclofen administration, and the enhanced effect lasts for long time. Lind et al. [5] reported that intrathecal baclofen enhances the effect of spinal cord stimulation on neuropathic pain. When they gave intrathecal baclofen to individuals with an SCS, pain reduction was significantly enhanced (from a mean VAS of 76 to 15 with $75 \mu \mathrm{g}$ baclofen).

Oral baclofen rarely controls dystonia in CRPS because it has a poor ability to cross the blood brain barrier. On the other hand, intrathecal baclofen results in greater therapeutic efficacy concentrated at the site of action in the spinal cord $[11,12]$. Patients should be screened to evaluate responsiveness to ITB therapy so that only suitable patients are selected, using a single or continuous infusion [13]. However, some patients on ITB do not experience satisfactory therapeutic effects despite good trial effectiveness of ITB because the implant fails to reach the target region of the spinal cord and the infusion system is very slow. To improve the success of ITB implantation, the location of the tip of the catheter in the in- 
trathecal pump infusion system relative to the targeted spinal cord segments may be critical to ensure the spread of drugs away from the catheter tip.

We assumed that the tip of the intrathecal catheter should be placed near the spinal site of action of baclofen in accordance with the sweet spot of the SCS. In our case, the catheter tip was placed in the ipsilateral dorsal subarachnoid space at same level as the sweet spot of the SCS lead, which provided adequate paresthesia covering the entire painful region. Highly concentrated baclofen should be used to fill the pump, because less concentrated baclofen in a larger volume causes patients discomfort stemming from the need to refill it more frequently. The volume of drug that is administered via an intrathecal drug pump is extremely small when highly concentrated baclofen is used. In our cases, the intrathecal pump infused at a very slow-rate, $0.048 \mathrm{ml} /$ day and 0.067 $\mathrm{ml} /$ day. Bernards reported that spread of baclofen within the cerebrospinal fluid (CSF) and spinal cord parenchyma is very limited rostrocaudally and circumferentially from the site of administration with very low-rate chronic infusion in pigs [14]. Therefore, pain reduction may not occur if the intrathecal catheter is not properly located. When the intrathecal catheter is placed in accordance with the patient's pain region, the extent of pain reduction will increase. Additionally, it may well be important to place the tip of the catheter posteriorly to reach the ipsilateral dorsal horn and regulate pain transmission. In this case, the patient had already been implanted with an SCS, so we determined the location of the tip of intrathecal catheter consistent with the area of the spinal cord that generated optimal stimuli from the SCS.

The optimal location of the tip of the intrathecal catheter was not determined according to the painful region in previous studies. Jose et al. [15] suggested that drug features, CSF dynamics, and patient characteristics should be considered when determining the location of the intrathecal catheter. We suggest that the spinal level of the SCS is a helpful factor when determining the positioning of the tip of the intrathecal catheter. Further studies are needed to determine the most suitable location for the tip of the intrathecal catheter.

There are various complications and side effects of the implantation. Surgical complications include operation-related infections, seroma, and abscess formation at the surgical site and epidural hematoma and abscess. Also, in our case, severe seroma occurred in the pump pocket. This could have been prevented by careful hemostasis, minimal pocket size, and drainage. Mechanical pump failure is rare and good anchoring avoids migration and torsion of the catheter. Baclofenrelated complications, which include drowsiness, headache, confusion, hypotension, constipation, nausea, increased urinary frequency, and sexual dysfunction, can occur at therapeutic doses. An overdose of intrathecal baclofen can be lifethreatening. This can occur in the setting of improper pump refill or accidently injecting medication into the side port [4].

In conclusion, this case report suggests that intrathecal baclofen administration provides satisfactory pain relief in CRPS patients with chronic intractable neuropathic pain not improved by an SCS.

\section{REFERENCES}

1. Merskey H, Bogduk N. Classification of chronic pain: descriptions of chronic pain syndromes and definitions of pain terms. 2nd ed. Seattle, IASP Press. 1994, pp 40-3.

2. Wilson PR, Yaksh TL. Baclofen is antinociceptive in the spinal intrathecal space of animals. Eur J Pharmacol 1978; 51: 323-30.

3. Zuniga RE, Schlicht CR, Abram SE. Intrathecal baclofen is analgesic in patients with chronic pain. Anesthesiology 2000; 92: 87680.

4. Slonimski M, Abram SE, Zuniga RE. Intrathecal baclofen in pain management. Reg Anesth Pain Med 2004; 29: 269-76.

5. Lind G, Meyerson BA, Winter J, Linderoth B. Intrathecal baclofen as adjuvant therapy to enhance the effect of spinal cord stimulation in neuropathic pain: a pilot study. Eur J Pain 2004; 8: 377-83.

6. Marinus J, Moseley GL, Birklein F, Baron R, Maihöfner C, Kingery WS, et al. Clinical features and pathophysiology of complex regional pain syndrome. Lancet Neurol 2011; 10: 637-48.

7. Geurts JW, Smits H, Kemler MA, Brunner F, Kessels AG, van Kleef M. Spinal cord stimulation for complex regional pain syndrome type I: a prospective cohort study with long-term followup. Neuromodulation 2013; 16: 523-9.

8. Kumar K, Rizvi S, Bnurs SB. Spinal cord stimulation is effective in management of complex regional pain syndrome I: fact or fiction. Neurosurgery 2011; 69: 566-78.

9. Hayek SM, Deer TR, Pope JE, Panchal SJ, Patel VB. Intrathecal therapy for cancer and non-cancer pain. Pain Physician 2011; 14: 219-48.

10. Penn RD, Kroin JS. Continuous intrathecal baclofen for severe spasticity. Lancet 1985; 2: 125-7.

11. van Hilten BJ, van de Beek WJ, Hoff JI, Voormolen JH, Delhaas EM. Intrathecal baclofen for the treatment of dystonia in patients 
with reflex sympathetic dystrophy. N Engl J Med 2000; 343: 62530.

12. van Rijn MA, Munts AG, Marinus J, Voormolen JH, de Boer KS, Teepe-Twiss IM, et al. Intrathecal baclofen for dystonia of complex regional pain syndrome. Pain 2009; 143: 41-7.

13. van der Plas AA, Marinus J, Eldabe S, Buchser E, van Hilten JJ. The lack of efficacy of different infusion rates of intrathecal baclofen in complex regional pain syndrome: a randomized, double-blind, crossover study. Pain Med 2011; 12: 459-65.

14. Bernards CM. Cerebrospinal fluid and spinal cord distribution of baclofen and bupivacaine during slow intrathecal infusion in pigs. Anesthesiology 2006; 105: 169-78.

15. Jose de A, Luciano P, Vicente V, Juan Marcos AS, Gustavo FC. Role of catheter's position for final results in intrathecal drug delivery. Analysis Based on CSF Dynamics and Specific Drugs Profiles. Korean J Pain 2013; 26: 336-46. 